\title{
Sopra certe classi di connessioni lineari.
}

\author{
Memoria di Stanistao Golab (Cracovia).
}

L'oggetto della seguente nota è l'esposizione di una serie di teoremi ammessi da una classe di connessioni lineari. Queste considerazioni hanno avuto origine dalla generalizzazione di una affermazione del sig. G. CorbeLLINI (1). Ho assunto le notazioni che si trovano nel libro di J. A. SoHouten, Der Ricci-Kalküll $\left(^{(}\right)$.

1. Sia dato nello spazio $n$-dimensionale $X_{n}$ il sistema di $n$ congruenze indipendenti di curve. Diciamo che questo sistema è olonomo se può essere stabilito in ogni punto $\xi$ dello spazio $X_{n}$ un sistema di riferimento $e^{\nu}\left({ }^{3}\right)$ (composto di $n$ vettori controvarianti) tale, che i vettori del detto sistema siano tangenti alle curve delle congruenze e formino un sistema olonomo nel senso ordinario $\left.{ }^{4}\right)$. Il sistema di riferimento composto dei vettori ${\underset{i}{v}}^{v}$ verrà indicato con (i). La condizione necessaria e sufficiente per l'olonomia del sistema (i) è :

$$
\partial_{[\mu} e_{\lambda]}^{k}=0 \quad(k=1, \ldots, \mathbf{n}),
$$

(1) G. Corbellin, Di una classe di varietà caratterizate per mezzo del parallelismo, "Rend. dei Lincei ", Vol. IV, 1926, ser. 6, p. 92-99. Cfr. anche E. BortolotTi, Reti di Cebiceff e sistemi coningati nelle $V_{\mathrm{n}}$ riemanniane, "Rend. dei Lincei ", Vol. V, ser. 6 (1927), p. $741-747$.

(1) J. A. Schouten, Der Ricci-Kalkül, Berlin, Springer, 1924. Quanto conceme le posteriori correzioni e semplificazioni, può trovarsi sistematicamente raccolto nel mio lavoro, Ueber verallyemeinerte projektive Geometvie, «Prace matematyczno-fizycane 》, Warszawa, 1930, T. 37, p. 91-153.

(3) Gli indici correnti dell' alfabeto greco percomono la serie $1, \ldots, n$, gli indici dell'alfabeto latino, invece la serie: $1, \ldots, n$. I valori dei simboli $1, \ldots, n$ debbono essere considerati come totalmente diversi dai valori $1, \ldots, n$.

( $\left.{ }^{4}\right)$ La letteratura concernente le ricerche suj sistemi anolononi nella geometria è citata nel lavoro di J. A. Schouten, Ueber nichtholonome Uebertragungen in einer $\mathrm{L}_{\mathrm{n}}$, Mathe. matische Zeitschrift ", 30, 1929, p. 149-172. 
dove il sistema $e_{j}^{k}$ dei vettori covarianti ò reciproco al sistema $e_{i}(j)$ e $\partial_{k}$ vale per $\partial / \partial \xi^{\mu}$. Se il sistema $(i)$ è dato per mezzo di arbitrari vettori tangenti $\underset{i}{p^{\nu}}$, allora le condizioni necessarie e sufficienti per l'olonomia sono le seguenti:

$$
{\stackrel{k}{k}{ }_{[\nu} \partial_{\mu}{ }_{\mu}^{k} p_{\lambda]}=0\left({ }^{6}\right)}_{1} \quad(k=1, \ldots, \mathrm{n}),
$$

dove $p_{\lambda}^{k}$ è di nuovo il sistema reciproco al sistema $p_{i}^{\nu}$. Quando la varietà $X_{n}$ è dotata di una connessione affine (lineare e simmetrica) ed in questa $\nabla$ significa la derivazione covariante, allora la condizione d'olonomia può essere scritta nella seguente forma, invariante rispetto alle trasformazioni di coordinate:

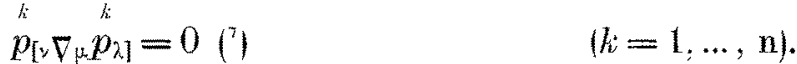

2. Lo spazio $X_{n}$ sia dotato di ana connessione lineare, generalmente non simmetrica, di parametri $\Gamma_{\lambda \mu}^{\prime}$. Per comodita introduciamo il seguente breve modo di esprimersi. Se è data una fimiglia di curve ad uno o più parametri, ed oltre a ciò una curva $L$ contenuta nella varietà luogo dei punti delle curve della nostra famiglia, allora l'espressione, che la data famiglia è parallela, nel senso del parallelismo generato dalla connessione, lungo la curva $L$, vuol dire che sussiste il fatto seguente: Se in un punto qualunque $\xi$ della curva $L$ prendiamo un vettore controvariante tangente a una curva della nostra famiglia e lo spostiamo parallelamente lungo la curva $L$, otteniamo sempre un vettore tangente a una curva della famiglia.

Introduciamo adesso le due seguenti definizioni:

Definizione I. - Diciamo che la connessione appartiene alla classe $(C)$ - o più in breve, che essa ha la proprietà $(C)$-, se esiste un sistema (i)

(j) Il sistema reciproco è è univocamente delinito per mezzo del sistema di equazioni

dove $A_{\grave{\alpha}}^{*} \grave{c}$ l'affinore-unità.

$$
\stackrel{\substack{k \\ e^{v}}}{h}=A_{\lambda}^{v}
$$

(6) Cfr. T. Levi-Grvita, The absolute Differential Caleulus, London, 1927, p. $26-29$ come pure G. Conbellini, Sopra $i$ sistemi di coorlinate di una varietà qualunque. "Rend. dei Lincei ", Vol. 32, 1923, p. 112-11t.

(a) Per le connessioni lineari generali (non-simmetriche) la condizione d'olonomia si presenta nella forma un po più complicata:

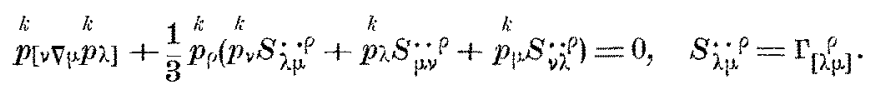


di $n$ congruenze indipendenti tale che ciascuna delle congruenze $[i]\left(^{8}\right)$ è parallela lungo ogni curva della congruenza $[j]$, per $j \neq i\left({ }^{\circ}\right)$.

Definizione II. - Diciamo che la connessione appartiene alla classe $(D)$, 0 più in breve, che essa ha la proprietà $(D)$, se esiste un sistema (i) di $n$ congruenze indipendenti tale che ciascuna delle congruenze [i] è parallela lungo ogni curva di una qualunque congruenza $[j]$.

$\mathrm{Si}$ vede subito che ogni comessione della classe $(D)$ appartiene alla classe (C), ma non reciprocamente. La definizione II esprime che la connes. sione ha la proprietà $(C)$ ed oltre a ciò, che le curve di ogni congruenza sono geodetiche. E chiaro che dal fatto, che una data connessione appartiene alla classe $(C)$ e che non tutte le congruenze, delle quali si parla nella de. finizione, sono geodetiche, non si può dedurre, che la connessione non ap. partiene alla classe $(D)$.

Indicheremo le condizioni, alle quali debbono soddisfare i parametri $\Gamma_{\lambda, \mu}$, affinchè la connessione abbia la proprietà $(C)$ o risp. (D). Queste condizioni non hanno però forma intrinseca. Valgono soltanto nel sistema (i), dato per mezzo dei vettori $p_{i}^{*}$, tangenti alle congruenze. La trasformazione di queste condizioni a forma intrinseca ci sembra un problema molto difficile.

Supponiamo che la connessione appartenga alla classe $(C)$ o risp. $(D)$, e denotiamo con $\mathrm{r}_{i j}^{k}$ i parametri della connessione riferiti al sistema $(i)$, dato per mezzo dei vettori $p_{i}^{v}$. Siano $[r],[s]$ due delle $n$ congruenze, menzionate nella definizione. Nel caso $(C)$ si suppone $r \neq s$, nel caso (D) può anche essere $r=s$. Nel punto $\xi$ arbitrario prendiamo un vettore tangente alla curva della congruenza $[r]$ e del resto arbitrario. Poichè - e questo è facile a dimostrare - la proprietà espressa al principio del $\S 2$ è soddisfatta da ogni vettore tangente, se è soddisfatta da uno, allora basterà prendere come punto di partenza il vettore $p_{r}{ }^{\nu}$. Le componenti di questo vettore $\underset{r}{p^{i}}$ rispetto al si. stema (i) sono uguali a:

$$
p_{r}^{k} \pm \delta_{r}^{k}\left({ }^{10}\right)
$$

(8) Il segno [i] vuol denotare, a differenza del segno (i), quella delle congruenze del sistema $(i)$, della quale il numero ordinale è $i$.

(9) Appunto le connessioni della classe (C) sono state introdotte nella geometria riemannian: da G. Corbercint nel lavoro citato sotto (1). E. Bortorotm chiama in questo caso il sistema $(i)$ rete di Cebiceff.

${ }^{(10)}$ Le $p^{p}$ sono le componenti del vettore menzionato nel sistema (v) appartenente alle coordinate $\xi^{y}$. Il sistema (i), dato per mezzo dei sistemi locali, pnò essere anolonomo e può non appartenere a nessun sistema di coordinate $\xi^{i}$. Cfr. J. A. Scrovten, 1. c. $\left(^{4}\right)$.

Il segno * vaol significare che l'equazione non ha il carattere intrinseco, ma vale sol- 
Dopo lo spostamento parallelo di questo vettore lungo la curva della congruenza $[s]$ le componenti dell' elemento lineare dello spostamento essendo $(d \xi)^{k}$ - le nuove componenti saranno

$$
\bar{p}_{r}^{k}=p_{r}^{k}-\Gamma_{i j}^{k} p_{r}^{i}(d \xi)^{j}
$$

Tenendo conto che conformemente alla definizione

$$
(d \xi)^{k}=p_{s}^{k} d t
$$

e che le equazioni (4) valgono per $k, r$ arbitrari, otteniamo

$$
\bar{p}_{r}^{k} \triangleq \delta_{r}^{k}-\Gamma_{r s}^{k} d t
$$

Poichè il vettore $\bar{p}_{r}^{h}$ deve restare tangente alla congruenza $[r]$, abbiamo la relazione

$$
\bar{p}^{k} \triangleq \propto \delta_{r}^{k}
$$

dove $x$ è un certo coefficiente. Dalle (7) e (8) risulta, che

$$
\Gamma_{r s}^{k}=0 \text { per } k \neq r \text {. }
$$

Le equazioni (7) e (8) per $k=r$ non forniranno niente di nuovo. Per le connessioni della classe $(D)$ siamo allora condotti alle equazioni (9) come condizioni necessarie. Com'è evidente conseguenza di tutte le considerazioni precedenti, nel caso delle connessioni della classe $(C)$ otteniamo le equazioni:

$$
\Gamma_{r s}^{k} 0 \text { per } k \neq r \text { e } s \neq r
$$

Si può adesso verificare, con la massima facilità, che le relazioni $(10)$ o risp. (9) sono nello stesso tempo sufficienti affinchè la connessione lineare abbia la proprietà $(C)$ o risp. $(D)$.

Si paò anche dedurre, dalle considerazioni precedenti, che le condizioni (9) o risp. (10) sono invarianti rispetto ai cambiamenti di sistemi di riferimento che consistono in dilatazioni locali $\left({ }^{11}\right)$ (non soltanto però per tali

tanto nel supposto sistema di riferimento. $\delta_{r}^{*}$ denota - come sempre - il simbolo di KroNeCKer, uguale a 1 per $k=r$ e a 0 per $k \neq r$.

(11) Cfr. L. Branchi, Lezioni di grometria differenziale, Pisa, 1922, Vol. I, § 60, p. 155 e 156. Diciamo, che il sistema $e_{I}$ è derivato del sistema $\underset{\dot{q}}{e^{v}}$ per dilatazione locale, se $e^{\vee}=e_{l}^{e v}$, dove $\rho_{l}$ sono scalari. 
cambiamenti), cioè se con (I) denotiamo il sistema che è ottenuto dal sistema (i) mediante una dilatazione, allora le equazioni (9) o risp. (10) implicano le equazioni

(9a)

$$
\Gamma_{I J=x_{-}}^{K} 0 \quad \text { per } \quad K \neq I
$$

risp.

$(10 \mathrm{a})$

$$
\Gamma_{I J}^{K^{*}} \underline{*}_{0} \text { per } K \neq I \text { e } J \neq I .
$$

3. Per le connessioni affini (simmetriche) vale il seguente

Teorema. - Se la connessione affine appartiene alla classe $(C)$, allora il sistema di congruenze (i), menzionato nella definizione, è un sistema olonomo.

Dimostrazione. - Conservando le notazioni, abbiamo fra i parametri $T_{i j}^{k_{i}}$ nel sistema $(i)$ e i parametri $\Gamma_{\not \mu}^{\nu}$ nel sistema (v) la relazione

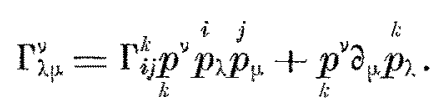

Moltiplicando i due membri dell' equazione precedente per $\stackrel{l}{p}_{v}$ e sommando rispetto all' indice $v$, otteniamo:

donde

$$
\partial_{\mu}^{l} p_{\lambda}=\stackrel{l}{p} p_{\nu} \Gamma_{\lambda \mu}^{\nu}-\Gamma_{i j}^{l} \stackrel{i}{p_{\lambda}} p_{\mu}
$$

Badando al fatto che la connessione è simmetrica e che il sistema (v) è olonomo, abbiamo:

dunque

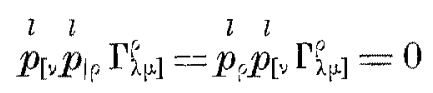

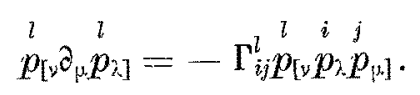

Osserviamo però che fra i trivettori

$$
\begin{aligned}
& l \quad i j \\
& p_{[\nu} p_{\lambda} p_{\mu]}
\end{aligned}
$$

sono diversi da zero (non degeneri) soltanto quelli pei quali $l \neq i, l \neq j, i \neq j$.

Dunque nella somma a destra della equazione (15) basta considerare soltanto i termini pei quali $l \neq i, l \neq j, i \neq j$. I coefficienti $l_{i j}^{k}$ di questi termini sono appunto uguali a zero in forza della (10), e in conseguenza

$$
\stackrel{l}{p_{[}, \partial_{j}, p_{\lambda]}}=0 \quad(l=1, \ldots, n),
$$

e questo esprime, che il sistema (i) delle congruenze è olonumo. 
Osserviamo che il teorema precedente non è vero in generale per le connessioni lineari. Esso è ovvio nel caso $n=2$, perchè in questo caso il trivettore (16) è sempre nullo.

4. Poichè le equazioni (9) o risp. (10) non hamno carattere intrinseco rispetto a tutte le trasformazioni di coordinate. si presenta la questione di decidere, quando sono dati i parametri $\Gamma$ della connessione in un qualunque sistema di riferimento, se la connessione appartenga alla classe $(D)$ o risp. $(C)$ o no. Questo problema appare nel caso generale molto difficile a risolvere. Noi mostriamo soltanto che per $n \geq 3$ la risposta è in generale negativa.

Fermiamoci nel caso particolare $n=2$, nel quale caso sussiste il seguente Teorema. - Per $n=2$ ogni connessione lineare gode della proprietà $(C)$. Drmostrazione. - Osserviamo anzitutto che per $n=2$ il sistema (10) consta, sia nel caso generale della connessione assimetrica, come nel caso della connessione affine (ciò̀ simmetrica), soltanto di due equazioni. Mostreremo che esiste un sistema di vettori $e_{i}^{y}$ tale che i parametri $\mathrm{T}_{i j}^{k}$, riferiti a questo sistema, soddisfano alle equazioni

$$
\Gamma_{21}^{1} * 0, \quad \Gamma_{12}^{2} * 0
$$

e questo - come gia sappiamo - basta per provare la nostra asserzione. Introduciamo a tale scopo le seguenti brevi notazioni:

$$
\left\{\begin{array}{c}
\sigma^{\prime}=-\Gamma_{\lambda \mu}^{\nu} A_{\nu}^{2} A_{1}^{\lambda} A_{2}^{\mu}, \quad \sigma^{\nu}=-\Gamma_{\lambda \mu \mu}^{\nu} A_{\nu}^{1} A_{1}^{\lambda} A_{2}^{\mu} \\
A_{\nu}^{k}=\frac{\partial \xi^{k}}{\partial \xi_{\nu}^{\nu}}, \quad A_{*}^{\nu}=\frac{\partial \xi^{\nu}}{\partial \xi^{k}},
\end{array}\right.
$$

e consideriamo il seguente sistema d'equazioni a derivate parziali del secondo ordine :

$$
\partial_{1} A_{2}^{v}=\sigma^{k} A_{k}^{y} \quad(v=1,2) \quad\left({ }^{12}\right)
$$

Il sistema precedente è un sistema di due equazioni parziali con due funzioni incognite $\xi^{1}\left(\xi^{1}, \xi^{2}\right)$, $\xi^{2}\left(\xi^{1}, \xi^{2}\right)$, equazioni risolute rispetto alle derivate parziali del secondo ordine miste, e che nei secondi membri contengono i quozienti di due polinomi nelle derivate parziali del primo ordine. Segue manifestamente dalle equazioni (19) e (20) che i denominatori sono del quarto,

(12) Le equazioni (20) possono ('ssere chiamate equazioni generalizzate di SERvaNT. Cfr. L. Bianchi, Leaioni di geometria differenziale. (1922), Vol, I, p. 157 . 
i numeratori del secondo grado $\left({ }^{13}\right)$, i coefficienti sono funzioni note delle funzioni incognite $\xi^{\prime}$. Tale sistema - com' ̀ ben noto della teoria generale delle equazioni a derivate parziali - ammette sempre soluzioni la questione delle condizioni iniziali non ha alcun interesse per noi). Prendendo una delle soluzioni con jacobiano diverso da zero (e tali certamente ne esistono), otte. niamo nello stesso tempo un passaggio dall'originario sistema di coordinate $\xi^{*}$ a un nuovo sistema di coordinate $\xi^{k}$. Se calcoleremo i parametri della connessione $\Gamma_{i j}^{k}$ nel nuovo sistema $(k)$ per mezzo delle formule di tra. sformazione

$$
\Gamma_{i j}^{k}=\Gamma_{\lambda \mu}^{\nu} A_{y}^{k} A_{i}^{\lambda} A_{j}^{\mu}+A_{\lambda}^{k} \partial_{j} A_{i}^{\lambda}
$$

e terremo conto che sussistono le equazioni (20), otterremo immediatamente le relazioni (18).

Osservazione I. - Il teorema precedente non può essere esteso (neppure nel caso $n=2$ ) alle connessioni lineari della classe $(D)$. Esistono connessioni, anche simmetriche e riemanniane, che non appartengono alla elasse $(D)$. Come più semplice esempio, basta prendere una qualunque con. nessione riemanniana non-enclidea (cfr. il teorema del paragrafo seguente).

OSSERtAZIONE II. - Il teorema precedente non può essere generalizzato pel caso di un numero di dimensioni maggiore di 2. Infatti, supponiamo che sia $n \geq 3$. Nel caso $(C)$ il sistema (10) contiene $n(n-1)^{2}$ equazioni con $n^{2}$ funzioni incognite $p_{i}$. Siccome per $n \geq 3$ si ha $n(n-1)^{2}>n^{2}$, allora il si. stema non ha in generale soluzioni. Anche supponendo che la connessione sia affine (simmetrica) e presumendo l'esistenza di soluzioni olonome, il sistema (10) si riduce a $\frac{n^{2}(n-1)}{2}$ equazioni, ma d'altra parte ha $n$ funzioni incognite, in generale, quindi, non esisteranno soluzioni, perchè si ha $\frac{n^{2}(n-1)}{2}>n$ per $n \geq 3$.

Ossertazione III. - Siccome il sistema (9) contiene in sè come sottosistema il sistema (10), è chiaro che in generale per $n \geq 3$ la connessione lineare non appartiene alla classe $(D)$.

Per mostrare come siano restrettive le condizioni affinchè la connessione abbia la proprietà $(D)$. citiamo i seguenti teoremi.

(13) Se immaginiamo di esprimere le derivate parziali $A_{\gamma_{2}}^{k}$ ehe vengono introdotte nelle formule (19) per meazo delle derivate del sistema reciproco, le prine saranno funzioni razionali di queste ultime, e precisamente, frazioni col numeratore del primo, il denominatore del secondo grado. 
5. Teorema. - Se una connessione affine è una connessione riemanniana avente come $d s^{2}$ una forma definita positiva e se essa appartiene alla classe (D), allora essa è una connessione euclidea (secondo ScHoutwa: cioè. essa fa della $X_{n}$ uno spazio enclideo) $\left({ }^{14}\right)$.

Dimostrazrone. - Denotiamo con (i) il sistema nel quale sussistono le equazioni (9). Secondo il teorema del paragrafo 3 possiamo da capo supporre che il sistema sia olonomo. Dnnque i parametri $\Gamma_{i j}^{k}$ della connessione calcolati nel sistema (i) sono simmetrici rispetto ai indici $i, j$, e per conseguenza il sistema (9) nel caso considerato equivale al sistema

$$
\Gamma_{i j=0}^{k}=0 \text { per } k \neq i \quad 0 \quad k \neq j \quad 0 \quad i \neq j
$$

In altre parole: nel sistema considerato sono diversi da zero soltanto i parametri della forma

$$
\gamma_{j}=\Gamma_{j j}^{j} \quad \text { (non sommare!). }
$$

Vediamo quale influenza hanno le equazioni (22) su la forma delle componenti del tensore di curvatura. Denotandolo come usualmente, abbiamo

$$
R_{i j i}{ }^{k}=\partial_{j} \Gamma_{i l}^{k}-\partial_{l} \Gamma_{i j}^{k}+\Gamma_{m i j}^{k} \Gamma_{i l}^{m}-\Gamma_{m l l}^{k} \Gamma_{i j}^{m}
$$

Tenendo conto delle equazioni (22) e (23), constatiamo successivamente che:
a) $R_{i j i}{ }^{k}=0$ per $l, j, i, k \neq$
b) $R_{k, j i}{ }^{k} * 0$ per $k, j, i \neq$
e) $R_{l j k}{ }^{k}=0$ per $l, j, k \neq$
d) $R_{i j i}{ }^{k}=0$ per $i, j, k+$
e) $R_{i k i}^{*} \stackrel{*}{=} 0$ per $k \neq i$
f) $R_{i+k i k}^{k} \stackrel{*}{=}-\partial_{l} \gamma_{k} \quad$ per $\quad k \neq l$
Non sommare rispetto agli indici che si ripetono!

Da questa tabella e dalla emmisimmetria del tensore $R_{l j i}^{k}$ rispetto agli indici $l, j$ segue che sono eventualmente diverse da zero soltanto le componenti

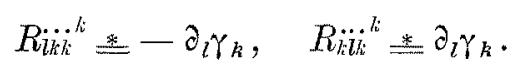

L'ipotesi che la connessione sia riemanniana equivale a supporre l'esistenza d'un tensore metrico $g_{i k}$ pel quale sussiste la proprietà :

$$
\partial_{j} g_{i k}=\Gamma_{i j}^{l} g_{l k}+\Gamma_{k i}^{l} g_{i l}
$$

(14) Questo teorema non ha niente in comune con quello contenuto nel $\$ 1$ del lavoro di E. BorToLotTr, gia menaionato in (1). 
onde, tenute presenti le (22), otteniamo

$$
\mathfrak{\partial}_{j} g_{i k}=\Gamma_{i j}^{i} g_{i k}+\Gamma_{k j}^{k} g_{i k}=g_{i k}\left(\Gamma_{i j}^{i}+\Gamma_{k j}^{k}\right)
$$

(non sommando nè rispetto a $i$ nè rispetto a $k$ !).

La formula precedente ci fornisce

$$
\begin{aligned}
& \text { a) } \partial_{j} g_{i k} \stackrel{*}{=} 0 \text { per } \quad i, j, k \neq \\
& \text { b) } \partial_{j} g_{i i}=0 \text { per } i \neq j \\
& \text { c) } \partial_{j} g_{i j} \stackrel{*}{*} \gamma_{j} g_{i j} \text { per } i \neq j \\
& \text { d) } \partial_{j} g_{j j}=2 \gamma_{j} g_{j j} .
\end{aligned}
$$

Da queste relazioni segue in particolare, che la componente $g_{i j}$ è funzione di due variabili soltanto $\xi^{i}$ e $\xi^{j}$. L' ultimo fatto può essere del resto dedotto dalla ipotesi meno restrittiva che la connessione sia riemanniana e possegga la proprietà $(C)$. (Cfr. su questo punto G. ConbellinI, 1. c., (1), \& 5). Dalla ipotesi che la forma differenziale $g_{i j} d \xi^{i} d \xi^{j}$ sia definita (positiva) segue in particolare che

$$
g_{j j}>0 \quad(j=1, \ldots, n) .
$$

Il nostro scopo è di dimostrare, che

$$
\partial_{j} \gamma_{i} \doteq 0 \quad \text { per } \quad i \neq j
$$

perchè allora, in virtù delle relazioni (25), svanirà identicamente il tensore di curvatura e questo - com'è ben noto - basta perchè la connessione sia euclidea. A tale scopo deriviamo $(29 \mathrm{~d}))$ rispetto a $\bar{\xi}^{i}$, snpponendo $i \neq j$ :

$$
\partial_{i j} g_{j j}=2 \gamma_{j} \partial_{i} g_{j i}+2 g_{j, j} \partial_{i} \gamma_{j} \text {. }
$$

Ora in virtù di $(29$ b) $)$ abbiamo:

$$
\partial_{i j} g_{j, j}=\partial_{j}\left(\partial_{i} g_{j j}\right) \stackrel{*}{=} 0 .
$$

Le (29 b)l, (33) e (32) dànno finalmente

$$
g_{i j} \partial_{i} \gamma_{j} \pm 0
$$

donde, in forza della (30), otteniamo la (31).

6. Teorema. - Se una connessione affine appartiene alla classe $(D)$ e gode della proprietà $R_{i k}=0\left(^{15}\right)$, allora essa è una connessione euclidea.

(15) Si ha $R_{i k}=R_{j i k}^{i j}$. 
Dinostrazlone. - Osserviamo che nel sistema $(i)$ abbiamo

$$
\left\{\begin{array}{l}
R_{\imath i} \stackrel{*}{=} 0 \\
R_{i k}=\partial_{i} \gamma_{k} \quad \text { per } \quad i \neq k .
\end{array}\right.
$$

Dalla relazione $R_{b k}=0$ segue allora che ha luogo l'equazione (31) e questo - come già sappiamo - ha per conseguenza la «enclideità » della con. nessione.

Osservazione. - Per $n=2$ il teorema precedente è ovvio. Per $n=3$ le cose vanno già diversamente. Bisogna ricordarsi che per $n=3$ la relazione $R_{i k}=0$ implica la relazione $R_{i j i}{ }^{k}=0$ soltanto nel caso in cui la con nessione è riemanniana, la quale ipotesi non è qui posta.

7. Teorema. - Se la connessione affine è proiettivamente euclidea $\left({ }^{16}\right)$ e appartiene alla classe $(D)$, allora è una connessione onclidea.

Drmostraztone. - Sia (i) - come prima - il sistema nel quale val. gono le equazioni (9). Denotiamo con $P_{i j i}^{k}$ il tensore di curvatura proiettiva di WEYL ( $\left.{ }^{17}\right)$. Abbiamo

$$
\left\{\begin{aligned}
P_{i j i}{ }^{k} & =R_{i j i}{ }^{k}+\frac{1}{n^{2}-1}\left\{(n-1) A_{i}{ }^{k}\left(R_{l j}-R_{j l}\right)-\right. \\
& \left.-A_{i}{ }^{k}\left(n R_{j_{i}}+R_{i j}\right)+1-A_{j}{ }^{k}\left(n R_{l i}+R_{i l}\right)\right\}
\end{aligned}\right.
$$

Tenendo conto delle (25) e (35), otteniamo successivamente:

$$
\begin{aligned}
& \text { a) } P \dddot{l i j}^{k}+0 \text { per } l, j, i, k \neq \\
& \text { b) } P_{h j i}^{* k} \stackrel{1}{=\frac{*}{1-n^{2}}}\left(\partial_{i} \gamma_{j}+n \partial_{j} \gamma_{i}\right) \text { per } k, j, i \neq \\
& \text { c) } P \dddot{j j k}^{k}=\frac{1}{n+1}\left(\partial_{\imath} \gamma_{j}-\partial_{j} \gamma_{l}\right) \text { per } k, l, j \neq \\
& \text { d) } \quad P_{i j i}^{k}=0 \text { per } i, j, k \neq \\
& \text { e) } P_{i k i} \stackrel{*}{=} 0 \text { per } k \neq i \\
& \text { f) } P i_{i k}^{k}=\frac{2-n}{n^{2}-1}\left(n \partial_{l} \gamma_{k}+\partial_{k} \gamma_{l}\right) \text { per } k \neq l \text {. }
\end{aligned}
$$

(Non sommare rispetto agdi indici che si ripetono!).

Siccome secondo l'ipotesi abbiamo

$$
P_{\not j i}^{k}=0
$$

(16) Vedi J. A. Schouten. Der Ricci-Kalkäl. p. 130.

(17) Vedi J. A. Schouten, Der Ricci-Kalkül, p. 131. 
segue dalla $(37 \mathrm{c})$

$$
\partial_{j} \gamma_{i} * \partial_{i} \gamma_{j}
$$

e l'ultima relazione confrontata per esempio con (37 b) fornisce

$$
\partial_{j} \gamma_{i} \pm 0 .
$$

Ne segue, in forza della $(25 \mathrm{f})$, whe

$$
R_{i j i}^{k}=0 \text {. }
$$

L'ultimo teorema è interessante, in quanto ne segue che la proprietà $(D)$ è la caratteristica che fra le connessioni affini proiettivamente enclidee distingue le connessioni euclidee. 